\title{
Drug Interactions for Low-Dose Inhaled Nemiralisib: A Case Study Integrating Modeling, In Vitro, and Clinical Investigations ${ }^{\text {[ }}$
}

\author{
Aarti Patel, Robert Wilson, Andrew W. Harrell, Kunal S. Taskar, Maxine Taylor, Helen Tracey, \\ Kylie Riddell, Alex Georgiou, Anthony P. Cahn, Miriam Marotti, and Edith M. Hessel
}

Drug Metabolism and Pharmacokinetics (A.P., A.W.H., K.S.T., M.T., H.T.) and Bioanalysis, Immunogenicity and Biomarkers (A.G.), GlaxoSmithKline R\&D, Ware, United Kingdom; RD Projects Clinical Platforms \& Sciences, GlaxoSmithKline R\&D, Stevenage, United Kingdom (R.W.); Global Clinical and Data Operations, GlaxoSmithKline R\&D, Ermington, Australia (K.R.); Discovery Medicine, GlaxoSmithKline, Stevenage, United Kingdom (A.P.C.); Safety and Medical Governance, GlaxoSmithKline R\&D, Stockley Park, Uxbridge, United Kingdom (M.M.); and Refractory Respiratory Inflammation Discovery Performance Unit, GlaxoSmithKline, Stevenage, United Kingdom (E.M.H.)

Received August 28, 2019; accepted January 27, 2020

\begin{abstract}
In vitro data for low-dose inhaled phosphoinositide 3-kinase delta inhibitor nemiralisib revealed that it was a substrate and a potent metabolism-dependent inhibitor of cytochrome P450 (P450) 3A4 and a P-glycoprotein (P-gp) substrate. An integrated in silico, in vitro, and clinical approach including a clinical drug interaction study as well as a bespoke physiologically based pharmacokinetic (PBPK) model was used to assess the drug-drug interaction (DDI) risk. Inhaled nemiralisib (100 $\mu \mathrm{g}$, single dose) was coadministered with itraconazole, a potent P4503A4/P-gp inhibitor, following 200 mg daily administrations for $\mathbf{1 0}$ days in $\mathbf{2 0}$ male healthy subjects. Systemic exposure to nemiralisib ( $A \cup C_{0-i n f}$ ) increased by 2.01-fold versus nemiralisib alone. To extrapolate the clinical data to other P4503A4 inhibitors, an inhaled PBPK model was developed using Simcyp software. Retrospective simulation of the victim risk showed good agreement between simulated and observed data $\left(A \cup C_{0-\text { inf }}\right.$ ratio 2.3 vs. 2.01, respectively). Prospective DDI simulations predicted a weak but manageable drug interaction when nemiralisib was coadministered with other P4503A4 inhibitors, such as the macrolides clarithromycin and erythromycin (simulated $\mathrm{AUC}_{0 \text {-inf }}$ ratio of 1.7), both common comedications in the
\end{abstract}

intended patient populations. PBPK and static mechanistic models were also used to predict a negligible perpetrator DDI effect for nemiralisib on other P4503A4 substrates, including midazolam (a sensitive probe substrate of P4503A4) and theophylline (a narrow therapeutic index drug and another common comedication). In summary, an integrated in silico, in vitro, and clinical approach including an inhalation PBPK model has successfully discharged any potential patient DDI risks in future nemiralisib clinical trials.

\section{SIGNIFICANCE STATEMENT}

This paper describes the integration of in silico, in vitro, and clinical data to successfully discharge potential drug-drug interaction risks for a low-dose inhaled drug. This work featured assessment of victim and perpetrator risks of drug transporters and cytochrome P450 enzymes, utilizing empirical and mechanistic approaches combined with clinical data (drug interaction and human absorption, metabolism, and pharmacokinetics) and physiologically based pharmacokinetic modeling approaches to facilitate bespoke risk assessment in target patient populations.
Introduction

Nemiralisib is a potent and highly selective inhaled phosphoinositide 3 kinase- $\delta$ inhibitor being investigated as an anti-inflammatory agent in respiratory diseases. In clinical studies, nemiralisib was generally welltolerated across a range of inhalation doses administered to both healthy volunteers and patients (Down et al., 2015; Cahn et al., 2017; Wilson et al., 2018; Begg et al., 2019). Conducting drug-drug interaction (DDI) studies is a required part of a drug's clinical development to assess the

Editorial support was provided by Kate Hollingworth of Continuous Improvement Ltd. and funded by GSK. This study was funded by GlaxoSmithKline.

A.W.H., R.W., K.R., A.G., and A.P.C. are GSK employees and hold GSK shares. A.P., K.S.T., M.T., H.T., M.M. are GSK employees. E.M.H. is a GSK employee and holds GSK shares and is on patents pertaining to the research discussed in the manuscript.

Availability of data and materials. Information on GSK's data sharing commitments and requesting access can be found at: https://www.clinicalstudydatarequest.com.

https://doi.org/10.1124/dmd.119.089003.

SThis article has supplemental material available at dmd.aspetjournals.org. potential risk that a drug may either cause a drug interaction as a perpetrator or be affected by another drug as a victim. For any drug interaction investigation, it is therefore important to consider both victim and perpetrator risks.

Bloomer et al. (2013) emphasize the importance of tailoring any drug interaction investigation to the common comedications in the intended patient populations and refer to diltiazem [potent P-glycoprotein (P-gp) inhibitor] and verapamil [moderate cytochrome P450 (P450) 3A4 inhibitor and potent $\mathrm{P}$-gp inhibitor] as frequently prescribed comedications in asthma and chronic obstructive pulmonary disease (COPD). Nemiralisib, however, is a drug under consideration for use in multiple respiratory indications, including bronchiectasis, and in patients experiencing frequent exacerbations in which common comedications additionally include theophylline (a sensitive narrow therapeutic index P4503A4 substrate) and the macrolide antibiotics erythromycin and clarithromycin (which are P4503A4 inhibitors). It was important, therefore, to investigate both victim and perpetrator interactions (particularly for P4503A4), assessments of which are guided by Food and Drug Administration (U.S. Department of Health and Human Services Food and Drug 
Administration, 2017a,b) and European Medicines Agency (2012). Clinically relevant victim drug interactions can occur even for lowdose inhaled molecules (Foisy et al., 2008), and thus clinical victim interaction studies are an expected feature of an inhalation clinical development plan. Following the completion of in vitro enzymology and transporter substrate packages (described herein), it was clear that the potential for P4503A4 and P-gp inhibitors to increase nemiralisib exposure required further investigation, particularly considering the comedication profile. The most effective way to study a victim drug interaction is through a clinical DDI study. In its clinical drug interaction guidance, U.S. Department of Health and Human Services Food and Drug Administration (2017a) recommend that a strong index inhibitor should be used to establish a worst-case interaction. Itraconazole is described as both a strong P450 inhibitor and potent $\mathrm{P}$-gp inhibitor, which was selected, therefore, as a suitable inhibitor for the study described herein.

U.S. Department of Health and Human Services Food and Drug Administration (2017b) outlines a tiered approach to investigate perpetrator DDIs, which starts with a simple application of basic equations, as described in Supplemental Table 1. If basic thresholds are triggered, then the investigation progresses to more complex static mechanistic models, which are detailed in Supplemental Table 2. The use of qualified PBPK models are an option provided in U.S. Department of Health and Human Services Food and Drug Administration (2017b) should the static mechanistic models also reveal a DDI risk. Should all these approaches indicate a DDI risk, then a clinical perpetrator DDI study may be necessary. Concern thresholds are rarely, if ever, triggered for low-dose inhaled molecules in which systemic exposure is extremely low. Despite this, it remains an expectation from some regulators that in vitro data are presented for inhaled molecules and for drug interaction risks to be discharged through application of the tiered approach, especially when comedications cannot be excluded in the desired patient population.

Using PBPK models to investigate drug interactions for oral or intravenous drugs is common practice, and PBPK models can be built in Simcyp, a commercially available PBPK software package and a proven methodology for assessing DDIs (Shebley et al., 2018). Herein, we describe a simple adaptation in Simcyp that enables an inhalation PBPK to model to be built and used to investigate drug interactions. To the authors' knowledge, an inhalation PBPK model has not previously been published for this purpose. Prior to developing and running prospective simulations with nemiralisib, the model was qualified using inhaled vilanterol, a P4503A4 substrate with a published P4503A4 clinical drug interaction study (Kempsford et al., 2013). The qualified PBPK model was then used to simulate the drug interactions as a victim following coadministration of strong and moderate P4503A4 inhibitors, such as clarithromycin and erythromycin (U.S. Department of Health and Human Services Food and Drug Administration, 2006) on nemiralisib exposure, or as a perpetrator (e.g., influence of nemiralisib on exposure to sensitive narrow therapeutic index P4503A4 substrates such as theophylline) (Tjia et al., 1996).

This paper presents the results of an integrated strategy for assessing the DDI risk for inhaled nemiralisib using in vitro, clinical, and in silico approaches, which considered both victim and perpetrator risks for both drug transporters as well as P450 enzymes.

\section{Materials and Methods}

\section{Materials}

Nemiralisib (Bis(6-(1H-indol-4-yl)-4-(5-\{[4-(propan-2-yl)piperazin-1yl]methyl $\}$-1,3-oxazol-2-yl)-1H-indazole) succinate) was supplied as a solid by GlaxoSmithKline (GSK) Chemical Registry (Stevenage, UK). The purity of the material was $>95 \%$ with a molecular weight of the bis succinate salt of 999.2. Glucose-6-phosphate, glucose-6-phosphate dehydrogenase, and $\beta$-nicotinamide adenine dinucleotide phosphate $(\mathrm{NADP}+)$ were supplied by Sigma Chemical Company (St. Louis, MO) or Corning Life Sciences (Woburn, MA). Benzylnirvanol, sulphaphenazole, furafylline, quinidine, digoxin, verapamil, estradiol glucuronide, rifampicin, cyclosporin A, p-Aminohippuric acid (PAH), probenecid, cimetidine, estrone sulfate, phenacetin, diclofenac (sodium salt), $\alpha$-naphthoflavone, furafylline, tamoxifen (citrate salt), Thio-triethylenephosphoramide, quercetin, S-fluoxetine, testosterone, nifedipine, and paroxetine hydrochloride were obtained from Sigma Chemical Company. Montelukast and tienilic acid were obtained from Cayman Chemical Company (Ann Arbor, MI). Efavirenz, S-mephenytoin, benzylnirvanol, R-bufuralol hydrochloride, midazolam, and gemfibrozil hydrochloride were supplied by Toronto Research Chemicals Inc. (Toronto, Ontario, Canada). Amadiaquine dihydrochloride dihydrate was supplied by Fluka Chemicals (St. Louis, MO). Nicotinamide adenine dinucleotide phosphate hydrate (NADPH) was supplied by MP Biomedical (Santa Ana, CA). Azamulin was supplied by the GSK compound bank (GSK, Harlow, UK) or Corning Life Sciences.

${ }^{3} \mathrm{H}$ Digoxin, ${ }^{14} \mathrm{C}$ Mannitol, ${ }^{3} \mathrm{H}$ Prazosin, ${ }^{3} \mathrm{H}$ Estradiol $17 \beta$-D-glucuronide, and ${ }^{3} \mathrm{H}$ Estrone sulfate were obtained from PerkinElmer Inc. (Waltham, MA). ${ }^{3} \mathrm{H}$ PAH was obtained from American Radiolabeled Chemicals (St. Louis, MO), Ko143 from Bio-Techne, and ${ }^{14} \mathrm{C}$ metformin from Curachem (Seol, Korea).

Pooled human liver microsomes (HLMs) prepared from a mixed gender pool of 150 or 200 donors were obtained from Corning or Xenotech LLC (Lenexa, KS), respectively. Human lung microsomes (HLuMs) were prepared from mixed gender pools of 10 human nonsmoker or smoker lungs (BioIVT, Baltimore, MD). Supersomes, containing individually overexpressed human P450 enzymes and derived from baculovirus-infected insect cells, and control Supersomes (lacking any native human P450 activity) were obtained from BD Gentest (Woburn, MA). Supersomes expressing P4502C8, P4502C9, P4502C19, P4503A4, P4502A6, $\mathrm{P} 4502 \mathrm{~B} 6, \mathrm{P} 4502 \mathrm{E} 1, \mathrm{P} 4502 \mathrm{~J} 2$, and $\mathrm{P} 4503 \mathrm{~A} 5$ coexpressed $\mathrm{P} 450$ reductase and cytochrome $b_{5}$, whereas Supersomes expressing P4501A2, P4502D6, and P4501A1 coexpressed P450 reductase only. Bactosomes expressing P4502A13 with coexpressed P450 reductase in Escherichia coli were obtained from Cypex Ltd. (Dundee, Scotland, UK). Supersomes and Bactosomes will be collectively referred to as recombinant P450 enzymes (rP450).

All other chemicals used in these investigations were reagent grade or higher and were obtained from standard commercial suppliers.

\section{Study Conduct of In Vitro Assays and Assessment of Risk}

The following work was conducted separate from the clinical drug interaction study: P450 and transporter phenotyping, P450 and transporter inhibition work, and development and application of the PBPK and mechanistic models. All of the work is reported in signed management-approved GSK reports, which are stored and available in GSK systems.

\footnotetext{
ABBREVIATIONS: AE, adverse event; $\mathrm{AUC}$, area under the plasma concentration time curve; $\mathrm{AUC}_{0 \text {-inf }}$, area under the plasma concentration time curve from time zero extrapolated to infinite time; BCRP, breast cancer resistance protein; $\mathrm{Cl}$, confidence interval; $\mathrm{C}_{\text {max }}$, maximum observed plasma concentration; COPD, chronic obstructive pulmonary disease; DDI, drug-drug interaction; ECG, electrocardiogram; GSK, GlaxoSmithKline; HLM, human liver microsome; HLuM, human lung microsome; $I_{50}$, half maximal inhibitory concentration; $I_{\text {max,u }}$, maximum unbound systemic concentration; $\mathrm{K}_{\mathrm{i}}$, inactivator concentration to achieve half-maximal inactivation rate; $\mathrm{k}_{\text {inact }}$, maximal rate constant of inactivation; MATE, multidrug and toxin extrusion; MDI, metabolism-dependent inhibition; MPPD, multiple path particle disposition; OAT, organic anion transporter; OATP, organic anion transporting polypeptide; OCT, organic cation transporter; MPPD, Multiple path particle dosimetry; P450, cytochrome P450; PAH, p-Aminohippuric acid; PBPK, physiologically based pharmacokinetic; P-gp, P-glycoprotein; PK, pharmacokinetic; R, ratio of victim AUC in the presence and absence of perpetrators (e.g. inhibitors), predicted with basic models; R1, R for reversible inhibition; R2, R for metabolism dependent inhibition; rP450, recombinant P450 enzymes; UPLC-LC/MS, ultraperformance liquid chromatography-liquid chromatography mass spectrometry.
} 


\section{In Vitro Investigation of Human Oxidative Enzymology of Nemiralisib}

The human P450 enzymes responsible for the oxidative metabolism of nemiralisib were investigated using a combination of in vitro incubation approaches in HLMs and HLuM with and without the presence of selective P450 inhibitors and using recombinant $\mathrm{P} 450$ enzymes. Incubation times, protein concentration, and cytochrome $\mathrm{P} 450$ content were predetermined from initial incubations to establish linear conditions.

Duplicate nemiralisib incubations were performed with pooled HLMs (30 minutes) and pooled HLuMs (120 minutes) in a shaking water bath. Each incubation contained $0.5 \mu \mathrm{M}$ (or $5 \mu \mathrm{M}$, data on file) nemiralisib, potassium phosphate buffer $(50 \mathrm{mM}, \mathrm{pH} 7.4)$, and either 0.5 or $2 \mathrm{mg} / \mathrm{ml}$ microsomal protein in the liver or lung microsome incubations, respectively. Reactions were initiated by the addition of cofactor solution (an NADPH regenerating system containing a final concentration of approximately $0.44 \mathrm{mM}$ NADP,$+ 5.5 \mathrm{mM}$ glucose-6phosphate, and $1.2 \mathrm{U} / \mathrm{ml}$ glucose-6-phosphate dehydrogenase). Reactions were terminated by adding a volume of acetonitrile equal to the incubation volume. Inhibition of nemiralisib cytochrome P450 metabolism was investigated using HLMs with a method previously described (Lawrence et al., 2014). Incubations ( $0.5 \mu \mathrm{M}$ nemiralisib, $0.5 \mathrm{mg} / \mathrm{ml}$ microsomal protein, 30 minutes) were conducted in duplicate in the presence and absence of the following selective P450 inhibitors: azamulin (P4503A4), sulphaphenazole (P4502C19), quinidine (P4502D6), montelukast (P4502C8), benzylnirvanol (P4502C19), or furafylline (P4501A2).

Nemiralisib incubations were also performed with a range of human $\mathrm{rP} 450 \mathrm{~s}$ containing overexpressed individual P450 enzymes. Each incubation contained $0.5 \mu \mathrm{M}$ nemiralisib, an appropriate volume of potassium phosphate buffer (50 $\mathrm{mM}, \mathrm{pH}$ 7.4), and $\mathrm{rP} 450$ at one of the following concentrations (and incubation times): $300 \mathrm{pmol} / \mathrm{ml}$ [P4501A2, P4502A6, P4502A13, P4502B6, P4502C9, P4502C19, P4502D6, or P4502E1 (120 minutes)], $75 \mathrm{pmol} / \mathrm{ml}$ [P4501A1 orP4502C8 (60 minutes)], $50 \mathrm{pmol} / \mathrm{ml}$ P4502J2 (60 minutes), or $25 \mathrm{pmol} / \mathrm{ml}$ [P4503A4, P4503A5 (30 minutes)]. Reactions were initiated and terminated in a similar manner to that described above.

Control incubations were performed in the absence of each of microsomes, Cofactor, rP450 enzymes, and nemiralisib. Following centrifugation of incubations at $13,000 \mathrm{~g}$ for 3 minutes, the supernatants were analyzed by ultraperformance liquid chromatography-liquid chromatography mass spectrometry (UPLC-LC/ $\mathrm{MS}^{\mathrm{n}}$ ) detection. Quantification was conducted on UV peaks over 3\% region of interest integrated peaks only. Quantitation by UV detection was preferred over MS for a number of reasons as follows: nemiralisib and its metabolites have strong chromophores, metabolite standard was only available for one metabolite (M1), and preliminary work (not shown) indicated better linearity for UV response compared with MS response. Drug-related UV peaks were identified following comparison of control and test incubations. Each drug-related UV peak was then integrated and expressed as percent region of interest, from which a concentration was calculated with reference to the nominal target incubation concentration. At both concentrations $(0.5$ and $5 \mu \mathrm{M})$, the main metabolites were quantifiable.

Metabolite structural assignments were based on UPLC-LC/MS ${ }^{\mathrm{n}}$ and/or comparisons of metabolite identification by UPLC-LC/MS ${ }^{\mathrm{n}}$ from previous work and authentic metabolites, where available.

In Vitro Evaluation of Nemiralisib as an Inhibitor of Cytochrome P450 Enzymes: 1A2, 2B6, 2C8, 2C9, 2C19, 2D6, and 3A4

Incubation conditions for the assay of the enzyme activities P4501A2, 2B6, 2C8, 2C9, 2C19, 2D6, and P4503A4 are shown in Supplemental Table 3. All incubations were performed at the concentration of substrate that leads to halfmaximal velocity previously determined for each probe substrate, and incubation times and HLM concentrations used reflect linear rate incubation conditions for the probe substrate reactions. For both reversible inhibition and metabolismdependent inhibition (MDI) experiments, positive control incubations (replacing nemiralisib) with an appropriate concentration range of a known reversible or metabolism-dependent P450 inhibitor and control incubations without inhibitor (containing 1\% v/v methanol only) were performed for all P450s. All samples were centrifuged to compress the precipitated protein into a pellet, and supernatants were monitored for probe substrate metabolite formation using LC/MS-MS. Further analytical details and details of internal standards are shown in Supplemental Table 4. Rates of metabolite production at each concentration of nemiralisib or positive control inhibitor were expressed as a percentage of the mean uninhibited control rate for each assay and half maximal inhibitory concentration $\left(\mathrm{IC}_{50}\right)$ curves fitted from a log-transformation of inhibitor concentration versus percentage remaining metabolite formation at each inhibitor concentration. MDI was inferred from a decrease in $\mathrm{IC}_{50}$ on preincubation in the presence versus the absence of NADPH.

\section{Reversible and MDI of P4501A2, 2B6, 2C8, 2C9, 2C19, and p4502D6}

Preincubation reaction mixtures contained incubation mix (100 mM sodium buffer, pH 7.4 and HLM) and an aliquot of nemiralisib solution in methanol, with and without NADPH (1 mM final concentration) for MDI and reversible inhibition, respectively. Triplicate incubations were prepared with each concentration of nemiralisib. After preincubation $\left(37^{\circ} \mathrm{C}, 30\right.$ minutes $)$, probe substrate was added to the reversible inhibition preincubation samples prior to the immediate initiation of reaction by addition of NADPH $(1 \mathrm{mM}$ final concentration). After preincubation $\left(37^{\circ} \mathrm{C}, 30\right.$ minutes) including $\mathrm{NADPH}$, reactions were initiated in the MDI preincubation samples by addition of probe substrate. Final incubation concentrations of nemiralisib were $0.0875,0.292$, $0.972,3.24,10.8,36$, and $120 \mu \mathrm{M}$. Reactions were terminated after the appropriate incubation time by the addition of $100 \mu$ l of acetonitrile containing a suitable internal standard.

\section{Reversible and MDI of P4503A4}

Preincubation reaction mixtures for P4503A4 contained 0, 0.03, 0.1, 0.3, $1.0,3.0,10$, and $30 \mu \mathrm{M}$ nemiralisib and microsomal protein in $100 \mathrm{mM}$ potassium phosphate buffer ( $\mathrm{pH} 7.4$ ), with and without an NADPHregenerating system (1.3 mM NADP,$+ 3.3 \mathrm{mM}$ glucose-6-phosphate, $0.4 \mathrm{U} / \mathrm{ml}$ glucose-6-phosphate dehydrogenase, and $3.3 \mathrm{mM}$ magnesium chloride). After 30 minutes of preincubation time at $37^{\circ} \mathrm{C}$, each preincubation reaction was diluted 1 in 10 by transferring an aliquot into a prewarmed secondary reaction mixture containing NADPH-regenerating system and one concentration of probe substrate in $100 \mathrm{mM}$ potassium phosphate buffer (pH 7.4). Reactions were incubated at $37^{\circ} \mathrm{C}$ for an appropriate incubation time, stopped by addition of $100 \mu \mathrm{l} 0.1 \%$ formic acid in acetonitrile containing a suitable internal standard, and placed on ice.

\section{Determination of the MDI Kinetics}

Inactivation incubations with nemiralisib were performed in a 96-well plate heated at $37^{\circ} \mathrm{C}$. Preincubation mixtures contained $100 \mathrm{mM}$ sodium or potassium phosphate buffer, pH 7.4 (P4501A2 and 2B6 and P4503A4, respectively), HLM, and various concentrations of nemiralisib solution in methanol. Inactivation reactions were initiated by the addition of NADPH [either as an aliquot of $10 \mathrm{mM}$ NADPH (P4501A2 and 2B6) or as an NADPH-regenerating system consisting of $1.3 \mathrm{mM}$ NADP+, $3.3 \mathrm{mM}$ glucose-6-phosphate, $0.4 \mathrm{U} / \mathrm{ml}$ glucose-6-phosphate dehydrogenase, and $3.3 \mathrm{mM}$ magnesium chloride (P4503A4)]. Final inactivation incubation concentrations of nemiralisib were $1.88,3.75,7.5,15,30,60$, and $120 \mu \mathrm{M}$ for P4501A2 and P4502B6 and 0, 0.1, 0.3, 1.0, 3.0, 10, 30. and $100 \mu \mathrm{M}$ for P4503A4. At multiple inactivation time points, aliquots of the inactivation reaction mixtures were withdrawn and diluted into a prewarmed $\left(37^{\circ} \mathrm{C}\right) \mathrm{P} 450$ specific activity assay incubation system containing NADPH or an NADPHregenerating system and the appropriate probe's substrate in phosphate buffer $(100 \mathrm{mM}, \mathrm{pH}$ 7.4). This activity assay mixture was incubated for an additional $\mathrm{P} 450$-specific incubation time. Reactions were terminated by the addition of stop solution containing an appropriate internal standard. Incubation details can be found in Supplemental Table 5. Positive control incubations (replacing nemiralisib with appropriate concentrations of furafylline for P4501 A2, thiotriethylenephosphoramide for P4502B6, or azamulin for P4503A4) and control incubations without inhibitor (containing $1 \% \mathrm{v} / \mathrm{v}$ methanol only) were also performed.

All samples were centrifuged to compress the precipitated protein into a pellet, and supernatants were monitored for probe substrate metabolite formation using LC/MS-MS. The natural logarithm of the residual activity (corrected for loss of activity observed over time in the absence of nemiralisib) was plotted against the preincubation time for each concentration of nemiralisib. The first-order rate constant for inactivation was estimated from the linear portion of the curves, and inactivation parameters [nemiralisib (inactivator) concentration required to achieve half-maximal inactivation rate $\left(\mathrm{K}_{\mathrm{i}}\right)$ and maximal rate constant of inactivation $\left(\mathrm{k}_{\text {inact }}\right)$ ] were determined using nonlinear regression analysis of the generated apparent rate constants at each concentration of nemiralisib. 


\section{Inhibition of Transporters by Nemiralisib}

An in vitro study to determine the inhibitory potency of nemiralisib against P-gp, breast cancer resistance protein (BCRP), organic anion transporting polypeptide 1B1 (OATP1B1), OATP1B3, OAT1, OAT3, organic cation transporter 2 (OCT2), multidrug and toxin extrusion 1 (MATE1), and MATE2-K, using porcine kidney epithelial cell line stably expressing P-gp or BCRP and human embryonic kidney mammalian cells stably expressing OATP1B1, OATP1B3, OAT1, OAT3, OCT2, MATE1, or MATE2-K and control cells, was conducted. Cell monolayers were preincubated at $37^{\circ} \mathrm{C}$ with buffer alone and buffer containing nemiralisib (0.0003-10 $\mu \mathrm{M}$ for P-gp and BCRP, and 0.001-30 $\mu \mathrm{M}$ for OATP1B1, OATP1B3, OAT1, OAT3, OCT2, MATE1, and MATE2-K). After preincubation was completed, cells were incubated in triplicate with buffer containing probe substrate, $1 \mu \mathrm{M}\left[{ }^{3} \mathrm{H}\right]$ digoxin (P-gp), $0.01 \mu \mathrm{M}\left[{ }^{3} \mathrm{H}\right]$ prazosin (BCRP), $0.05 \mu \mathrm{M}\left[{ }^{3} \mathrm{H}\right]$ Estradiol $17 \beta$-D-glucuronide (OATP1B1 and OATP1B3), $1 \mu \mathrm{M}\left[{ }^{3} \mathrm{H}\right] \mathrm{PAH}$ (OAT1), $0.05 \mu \mathrm{M}\left[{ }^{3} \mathrm{H}\right] \mathrm{ES}$ (OAT3), or $10 \mu \mathrm{M}\left[{ }^{14} \mathrm{C}\right]$ metformin (OCT2, MATE1, and MATE2-K) and appropriate amount of nemiralisib. Verapamil (P-gp), Ko143 (BCRP), cyclosporine A (OATP1B1 and OATP1B3), probenecid (OAT1 and OAT3), and cimetidine (OCT2, MATE1, and MATE2-K) were included as positive control inhibitors. Further details of the experimental conditions can be found in Supplemental Table 6.

\section{Clinical DDI Study Design, Subjects, and Assessments}

Study Design. The DDI study was a phase I, single-center, open-label, crossover study in healthy male subjects conducted by Quintiles (Kansas) between January 2018 and March 2018 (GSK study number 206874; ClinicalTrials.gov registration number NCT03398421). The study was approved by the Chesapeake Institutional Review Board (Columbia, MD) and adhered to the Declaration of Helsinki, and written informed consent was obtained from each subject.

All subjects had a screening visit followed by two treatment periods, with a 14-day washout period between treatments (Fig. 1). Nemiralisib hemisuccinate salt was blended with lactose and magnesium stearate and packed in foil blisters containing $100 \mu \mathrm{g}$ free base per blister in the Ellipta device. In treatment period 1, subjects received a single oral-inhaled dose of nemiralisib, $100 \mu \mathrm{g}$ via the Ellipta inhaler on day 1, and blood samples for pharmacokinetic (PK) analysis were collected up to 120 hours postdose. In treatment period 2, subjects received 2 oral capsules of itraconazole (200 mg total dose; supplied by Amneal Pharmaceuticals LLC, Bridgewater, NJ; manufactured by Amneal Pharmaceuticals Pvt. Ltd., Ahmedabad, India) daily from days 1 to 10 and a single oral-inhaled dose of nemiralisib $100 \mu \mathrm{g}$ on day 5, 1 hour after itraconazole. Blood samples for PK analysis were collected on day 1 (predose and up to 12 hours postdose) and day 5 (predose and up to 24 hours postdose) for itraconazole and hydoxy-itraconazole and on day 5 (up to 144 hours postdose) for nemiralisib. This design was based on that reported by Ke et al. (2014).

Subjects. Healthy male subjects who were aged from 18 to 75 years, weighed $50 \mathrm{~kg}$ or more, and had a body mass index of $18-35 \mathrm{~kg} / \mathrm{m}^{2}$ and normal spirometry (both forced expiratory volume in 1 second and forced vital capacity $\geq 80 \%$ of predicted) were included. Subjects with any clinically significant disease (including abnormal liver function), abnormal blood pressure, or a history of alcohol or drug abuse and current smokers (or smoking history within previous 6 months and a total pack year history of $>5$ pack years) were excluded. Full eligibility criteria details are given on ClinicalTrials.gov (NCT03398421).

Pharmacokinetic Assessments. Blood samples were collected into tripotassium ethylenediaminetetraacetic acid tubes and centrifuged, and plasma was harvested and stored at $-20^{\circ} \mathrm{C}$ prior to shipment for analysis. Plasma samples were analyzed for nemiralisib (GSK, Philadelphia) using a validated analytical method based on protein precipitation using acetonitrile containing $\left[{ }^{2} \mathrm{H}_{7}\right]$ nemiralisib as an internal standard, followed by ultra-high performance liquid chromatography and TurboIonSpray tandem mass spectrometric detection. The lower limit of quantification was $5 \mathrm{pg} / \mathrm{ml}$ using a $50 \mu \mathrm{l}$ aliquot of human plasma with a higher limit of quantification of $1000 \mathrm{pg} / \mathrm{ml}$. Typically, a $10 \mu \mathrm{l}$ aliquot of extracted sample was injected onto an LC system consisting of a $50 \times 2.1 \mathrm{~mm}$ i.d. Waters Acquity BEH C18 $1.7 \mu \mathrm{m}$ column and a Waters Acquity UPLC system. Mobile phases of $10 \mathrm{mM}$ ammonium bicarbonate $(\mathrm{pH} 9)$ and acetonitrile at a flow rate of $0.8 \mathrm{ml} / \mathrm{min}$ and a column temperature of $60^{\circ} \mathrm{C}$ were used to elute nemiralisib at a typical retention time of 0.9 minutes. Analysis for itraconazole and hydroxy-itraconazole (Covance Laboratories Limited, Harrogate, UK) was performed using an analytical method based on solid phase extraction followed by LC/MS-MS analysis. The lower limit of quantification was $2 \mathrm{ng} / \mathrm{ml}$ using a $100 \mu \mathrm{l}$ aliquot of EDTA plasma. The higher limit of quantification was $1000 \mathrm{ng} / \mathrm{ml}$.

Safety Assessments. In treatment period 1, vital signs and 12-lead electrocardiograms (ECGs) were measured prenemiralisib dose on day 1 and at day 6 before discharge. In treatment period 2, vital signs/ECGs were measured on day 1 (predose) and 3 hours postdose (ECG only) and predose on days 2, 4, 6, 8, and 10. Routine laboratory tests were performed at day -1 for each treatment period and on day 6 (treatment period 1) and day 10 (treatment period 2). In treatment period 2, additional samples for clinical chemistry were collected predose on days 2, 4, 6, and 8. Adverse events (AEs) were monitored throughout the study.

Static Mechanistic Evaluation for Nemiralisib Effect on Theophylline Exposure. Theophylline is an oral bronchodilator commonly prescribed in the treatment of asthma and COPD. In vitro studies have shown P4501A2 to be the major enzyme responsible for the metabolism of theophylline with minor contributions from other P450 enzymes, including P4503A4 (Tjia et al., 1996). To allow safe dosing of theophylline with nemiralisib, a mechanistic-based drug interaction assessment was conducted using the P4503A4 inactivation kinetic parameters determined for nemiralisib.

The static mechanistic model, as described by the U.S. Department of Health and Human Services Food and Drug Administration (2017b), incorporates irreversible inhibition and contribution of intestinal metabolism for P4503A4 substrates, which were applied to estimate the magnitude of increase in theophylline exposure [area under the concentration time curve (AUC)] when coadministered with nemiralisib. As a conservative approach, the fraction of liver metabolism of theophylline by P4503A4 was set to 1 . Intestinal metabolism of theophylline was deemed negligible based on the minimal difference in AUC ratios observed in clinical intravenous and oral drug interaction studies with Verapamil (Sirmans et al., 1988; Stringer et al., 1992).

\section{PBPK Modeling to Predict Nemiralisib DDI}

PBPK Platform Qualification. A clinical DDI study between vilanterol and ketoconazole was simulated to qualify the use of the PBPK platform Simcyp (Version 17, Release 1; Simcyp Ltd., Sheffield, UK). Like nemiralisib, vilanterol is an inhaled drug with metabolism via P4503A4 the main clearance route in humans and was therefore deemed suitable for qualification of the prediction of DDI between an inhaled P4503A4 substrate and potent P4503A4 inhibitor to regulatory expectations. A clinical study following coadministration of repeat once-daily $400 \mathrm{mg}$ of ketoconazole and single dose $25 \mu \mathrm{g}$ inhaled vilanterol resulted in an average 1.9-fold increase in vilanterol systemic exposure (AUC) but negligible change in its maximum observed plasma concentration $\left(\mathrm{C}_{\max }\right)$. A PBPK model incorporating the mechanistic clearance pathway was built for vilanterol using Simcyp (a listing of model parameters is shown in Supplemental Table 7) and validated with actual clinical studies at 100 and $25 \mu \mathrm{g}$ inhaled vilanterol. Once validated, a DDI simulation was then run between vilanterol and ketoconazole (in-built file within Simcyp), and the extent of simulated DDI was compared with the actual clinical DDI study (Kempsford et al., 2013).

Nemiralisib Inhalation PBPK Model Development and Qualification. An intravenous mechanistic PBPK model was developed initially using the Simcyp absorption, distribution, metabolism, and excretion simulator. To develop the nemiralisib compound file, measured physicochemical properties (e.g., molecular weight, octanol:water partition coefficient, blood to plasma ratio, and plasma protein binding) were entered into the Simcyp software along with mean observed volume of distribution and intravenous clearance, using data from a previous clinical study (Harrell et al., 2019). A full listing of the nemiralisib model parameters are shown in Supplemental Table 8. Simulations were then conducted to predict and compare nemiralisib plasma exposure (AUC) and half-life after a $10 \mu \mathrm{g}$ (nominal dose) 15-minute intravenous infusion. Simcyp-simulated dosing regimens for studied scenarios were performed according to their actual clinical trial study design and delivered doses (Harrell et al., 2019; Wilson et al., 2019). To complete the intravenous PBPK model, the in vivo clearance was converted to enzymes kinetics using the retrograde calculator within Simcyp version 17. The mean P4503A4\% contribution used for the calculator was determined in vitro from recombinant expressed enzymes and HLM. The remaining non-P4503A4 clearance routes were combined under additional HLM clearance. The intravenous PBPK model was converted to an oral PBPK model using the first order oral absorption model within Simcyp, and the model was qualified by comparing simulated fraction absorbed and bioavailability with observed following a single oral dose of $800 \mu \mathrm{g}$ nemiralisib (Harrell et al., 2019). The final stage of the PBPK model build used the 


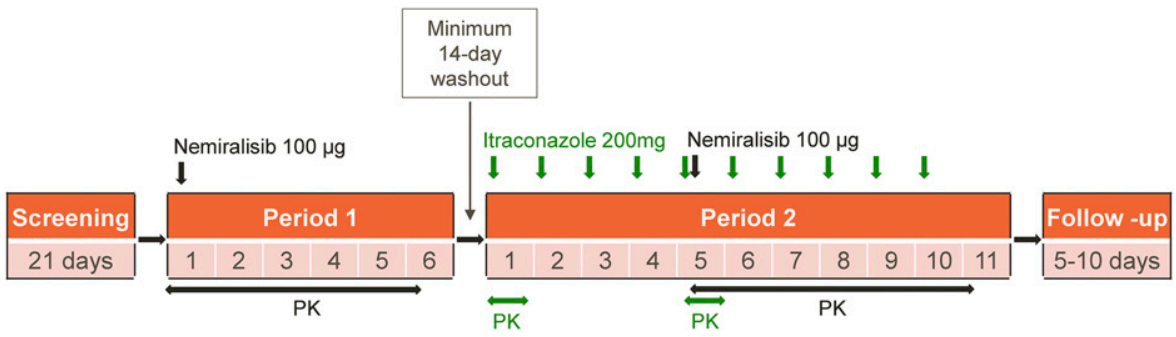

Fig. 1. Study schematic.

parameter estimation module to optimize the rate of absorption to the lung after an inhaled dose, using AUC data after a 1000- $\mu$ g dose (Harrell et al., 2019). The estimate of fraction absorbed in the lung was obtained from dosimetry data generated using a stand-alone computational model, Multiple-Path Particle Dosimetry (MPPD) version 2.92.5 (Applied Research Associates, Raleigh, NC).

The PBPK inhaled model was further qualified by comparing simulated data with plasma exposures of nemiralisib after a 500- $\mu \mathrm{g}$ dose (Wilson et al., 2019) and $100-\mu \mathrm{g}$ dose (clinical DDI study).

A retrospective simulation of the clinical DDI study between nemiralisib and itraconazole (NCT03398421 as described herein) was performed to evaluate whether Simcyp could accurately simulate this interaction based on the nemiralisib profile described.

\section{Nemiralisib Inhalation PBPK Model Application.}

Nemiralisib inhaled PBPK model victim simulations. To predict the effect of the macrolide inhibitors, clarithromycin and erythromycin, on the exposure of nemiralisib, drug interaction simulations were conducted using the clinical study designs from Gorski et al. (1998) and Olkkola et al. (1994).

Nemiralisib inhaled PBPK model perpetrator simulations. To predict the effect of nemiralisib on the exposure of the sensitive P4503A4 index substrate midazolam, a typical perpetrator drug clinical dosing regimen was simulated. To fully elucidate the effect of nemiralisib, repeat dose exposure profiles were simulated to steady state.

\section{Statistical Analysis}

For the in vitro investigation, statistical analysis was limited to the calculation of means when appropriate (Excel; Microsoft, Redmond, WA). Metabolite peaks were quantified by fractional conversion from parent observed on the chromatograms by UV area detection.

The sample size for the clinical DDI study was primarily based on feasibility. It was estimated, however, that with 16 subjects providing the relevant PK parameter data, the upper bound of the $90 \%$ confidence interval (CI) would be within approximately $22 \%$ of the point estimate and the lower bound within $18 \%$ for $\mathrm{AUC}_{0 \text {-inf. }}$ For $\mathrm{C}_{\mathrm{max}}$, the upper bound of the $90 \% \mathrm{CI}$ was estimated to be within approximately $23 \%$ of the point estimate and the lower bound within $19 \%$. Plasma concentration time data for nemiralisib and for itraconazole were analyzed by noncompartmental methods with WinNonlin Version 7.0, using the actual sampling times recorded during the study for derivation of PK parameters. For the PBPK modeling simulations, the statistical assessment for each set of data were performed by comparing the distribution of the simulated individuals to the distribution of the observed data.

\section{Application of Tiered Approach Guided by the Food and Drug Administration}

A tiered approach to investigating DDI, as described in U.S. Department of Health and Human Services Food and Drug Administration (2017b), was followed for nemiralisib. The Food and Drug Administration recommends an initial investigation using basic equations that essentially compare enzyme potency to free (unbound) drug systemic exposure (referred to as $I_{\max , \mathrm{u}}$ ) to generate values for $R_{1}$ (reversible inhibition), $R_{2}$ (metabolism-dependent inhibition), and $\mathrm{R}_{1}$ gut (reversible inhibition at the gut). Further details can be found in Supplemental Table 1. Further investigations are required if parameter thresholds are exceeded following the application of these equations $\left(\mathrm{R}_{1} \leq\right.$ $1.02, R_{2} \leq 1.25$, or $R_{1}$ gut $\leq 11$ ). As a result, the determination of $I_{\max , \mathrm{u}}$ is an important component of any perpetrator drug interaction potential consideration. For nemiralisib, $I_{\text {max,u }}$ was calculated assuming a pharmacological dose of $750 \mu \mathrm{g}$ (delivered as a dry powder in the intended clinical device by the inhalation route).
The steady-state plasma $\mathrm{C}_{\max }$ was assumed to be $3.6 \mathrm{ng} / \mathrm{ml}$, calculated from measured single-dose data (Wilson et al., 2019), multiplied by an accumulation ratio measured following repeated administrations (Wilson et al., 2018), and it was assumed that the free fraction in plasma was 0.021 (2.1\%) (unpublished data). As would be expected for a low-dose inhalation molecule, $I_{\max , u}$ was extremely low (calculated as $0.2 \mathrm{nM}$, using a free base molecular weight of 441).

\section{Results}

\section{In Vitro Oxidative Enzymology of Nemiralisib}

Incubation of $0.5 \mu \mathrm{M}$ nemiralisib, with the range of rP450 enzymes investigated together with the scaling of the expressed metabolite production to reflect amounts present in HLMs, indicated that the P450 enzyme predominantly responsible for metabolism of nemiralisib was P4503A4 (95\% contribution to metabolism; Table 1). P4501A2, $2 \mathrm{C} 8,2 \mathrm{C} 9,2 \mathrm{C} 19$, and 2D6 collectively contributed to $\sim 5 \%$ of hepatic metabolism of nemiralisib. Following the incubation of $0.5 \mu \mathrm{M}$ nemiralisib with HLMs and selective P450 inhibitors, the production of M1 (a major N-dealkylated metabolite) was predominantly inhibited by the P4503A4 inhibitor azamulin (55\%), followed by the P4502C8 inhibitor montelukast (15\%). As P4503A4\% contribution ranged from 55\% to 95\%, a mean contribution of $\sim 75 \%$ P4503A4 metabolism was used in the PBPK model. Nemiralisib was not metabolized by HLuMs. Because of the predominant contribution of P4503A4 metabolism of nemiralisib observed in vitro in liver, it was not unexpected that no metabolism in HLuMs was seen where P4503A4 expression in lung is negligible (Somers et al., 2007).

\section{In Vitro Inhibition of P450s in HLMs by Nemiralisib}

Nemiralisib inhibited the probe marker activity of P4501A2, 2B6, 2C8, 2C9, 2C19, 2D6, and 3A4 in HLMs, with $\mathrm{IC}_{50}$ values of 58.2, 44.4, $26.4,13.6,12.0,7.94$, and $>3 \mu \mathrm{M}$, respectively. Nemiralisib was an MDI of P4501A2, P4502B6, and P4503A4 with a decrease in $\mathrm{IC}_{50}$ value following a 30-minute preincubation with cofactor of 2.7, 1.9-fold, and $>7.9$ (highest fold change observed), respectively. MDI kinetic evaluation showed that nemiralisib was not an inactivator of P4501 A2. It was, however, an apparent inactivator of $\mathrm{P} 4502 \mathrm{~B} 6$ and $3 \mathrm{~A} 4$, with a $\mathrm{k}_{\text {inact }}$ of 0.014 and 0.034 minutes $^{-1}$ and $\mathrm{K}_{\mathrm{i}}$ of 2.0 and $4.2 \mu \mathrm{M}$, respectively (Table 2). The potential for increased exposure of the P4502B6 substrate Bupropion, on coadministration with nemiralisib, was estimated using

TABLE 1

Total percent contribution of individual P450 enzymes to nemiralisib metabolism

\begin{tabular}{lc}
\hline rP450 Enzyme & $\begin{array}{c}\text { \% Contribution to } \\
\text { Overall Metabolism }\end{array}$ \\
\hline P4501A2 & 0.9 \\
P4502C8 & 2.1 \\
P4502C9 & 1.8 \\
P4502C19 & $<0.1$ \\
P4502D6 & 0.3 \\
P4503A4 & 94.9
\end{tabular}

rP450, recombinant $\mathrm{P} 450$. 
TABLE 2

Quantitative DDI victim risk assessment for nemiralisib: P450 inhibition

\begin{tabular}{|c|c|c|c|c|}
\hline \multirow[b]{2}{*}{ Cytochrome P450 } & \multirow{2}{*}{$\begin{array}{l}\text { Fold Change in } \\
\qquad \mathrm{IC}_{50}{ }^{a}\end{array}$} & \multicolumn{2}{|c|}{ Inactivation Parameters } & \multirow{2}{*}{$\begin{array}{c}\text { Is a DDI Risk } \\
\text { Predicted? }\end{array}$} \\
\hline & & $\begin{array}{c}\mathrm{K}_{\mathrm{I}} \\
(\mu \mathrm{M})\end{array}$ & $\begin{array}{c}k_{\text {inact }} \\
\left(\min ^{-1}\right)\end{array}$ & \\
\hline $1 \mathrm{~A} 2$ & 2.7 & \multicolumn{2}{|c|}{$\begin{array}{l}\text { No inactivation of } \\
\text { P4501A2 }\end{array}$} & \\
\hline $2 \mathrm{~B} 6$ & 1.9 & 2.0 & 0.014 & $\mathrm{No}^{b}$ \\
\hline $\begin{array}{l}2 \mathrm{C} 8,2 \mathrm{C} 9,2 \mathrm{C} 19 \& \\
\text { 2D6 }\end{array}$ & $<1.5$ & & & \\
\hline $3 \mathrm{~A} 4$ & $>7.9^{c}$ & 4.2 & 0.034 & $\mathrm{No}^{d}$ \\
\hline
\end{tabular}

DDI, drug-drug interaction; $\mathrm{IC}_{50}$, half maximal inhibitory concentration; $\mathrm{K}_{\mathrm{i}}$, inactivator concentration to achieve half-maximal inactivation rate; $\mathrm{k}_{\text {inact }}$, maximal rate constant of inactivation.

${ }^{a} \mathrm{~A}$ less than 1.5 -fold change in $\mathrm{IC}_{50}$ is deemed a low risk of DDI, and no additional inactivation data were generated.

${ }^{b}$ Risk discharged using static mechanistic models described by U.S. Department of Health and Human Services Food and Drug Administration (2017b) and European Medicines Agency (2012).

${ }^{c}$ Worst case.

${ }^{d}$ Risk discharged using physiologically based pharmacokinetic modeling.

static mechanistic models. When surrogates of nemiralisib concentration were corrected for plasma protein binding, the maximum extrapolated drug interaction was a 1.0-fold change for bupropion, indicative of no anticipated change of their exposure because of P450 inhibition when coadministered with nemiralisib. A parallel assessment with the P4503A4 probe substrate midazolam suggested that nemiralisib may elicit a potential GI- mediated clinical DDI. To further confirm and quantify this risk, the PBPK model described herein was used to simulate nemiralisib dynamic profiles with midazolam.

\section{In Vitro Inhibition of Transporters by Nemiralisib}

Nemiralisib, at a concentration of $30 \mu \mathrm{M}$, decreased the P-gp-mediated transport of digoxin by $69 \%$ and inhibited BCRP-mediated transport of Prazosin by $74 \%$. Nemiralisib $(30 \mu \mathrm{M})$ inhibited OATP1B1 and 1B3mediated transport of estradiol glucuronide transport by $89 \%$ and $85 \%$, with resulting $\mathrm{IC}_{50}$ values 3.03 and 1.76 , respectively. Nemiralisib reduced transport of metformin via OCT2, MATE1, and MATE2-K by $98 \%, 99 \%$, and $100 \%$, leading to submicromolar $\mathrm{IC}_{50}$ values $(0.381,0.0260$, and $0.0545 \mu \mathrm{M}$, respectively). Nemiralisib did not inhibit drug transporters OAT1 and OAT3 at concentrations up to $30 \mu \mathrm{M}$. The inhibitory effects of nemiralisib and positive control inhibitors are summarized in Supplemental Table 9. Unpublished GSK data has indicated that nemiralisib is a substrate of P-gp and BCRP but not a substrate for OAT1B1, OAT1B3, and OCT1.

\section{Clinical DDI Study}

Demographic and Baseline Characteristics. Twenty healthy male subjects were enrolled and completed the study. Their mean (S.D.) age was 36 (12) years, and body mass index was $28(3.9) \mathrm{kg} / \mathrm{m}^{2}$.

Pharmacokinetic Data. The plasma PK profiles of inhaled nemiralisib were visually similar following single dose administration of nemiralisib and administration of nemiralisib with itraconazole, characterized by fast absorption (time to maximum observed plasma concentration of 0.7 and 0.9 hours, respectively) and $\mathrm{C}_{\max }$ occurring up to 2 hours postdose, followed by a rapid, then slower, decline in plasma concentrations (Fig. 2). Nemiralisib plasma concentrations were higher following nemiralisib dosed with itraconazole compared with nemiralisib alone. The adjusted geometric least squares mean ratios for $\mathrm{AUC}_{0 \text {-inf }}$ and $\mathrm{C}_{\max }$ demonstrated, respectively, that the systemic exposure of nemiralisib was $101 \%$ greater (an increase of 2.01 -fold) and maximum plasma concentration was $20 \%$ lower following coadministration versus nemiralisib alone (Table 3). The 90\% CIs for the geometric LS mean ratios fell entirely outside the standard equivalence limits of $0.80-1.25$ for these parameters, indicating that itraconazole had a clinically relevant effect on the systemic exposure of nemiralisib. Plasma exposures for itraconazole and hydroxy-itraconazole are shown in Supplemental Table 10 .

Safety. One AE of headache was reported following treatment with nemiralisib alone; AEs of upper respiratory tract infection (two events) and one each of hypoacusis and dry eye were reported in the nemiralisib with itraconazole arm. No serious AEs or AEs leading to withdrawal were reported. The event of hypoacusis was judged as related to itraconazole by the investigator, was mild in intensity, and was resolved after 2 days. There were no clinically significant changes in other safety parameters.

Static Mechanistic Evaluation for Nemiralisib Effect on Theophylline Exposure. The static mechanistic model to evaluate the impact of nemiralisib on theophylline showed that at clinically relevant concentrations, nemiralisib would not alter the systemic exposure of theophylline, with the highest potential fold change for theophylline exposure predicted to be $<1.2$ fold.

\section{Physiologically Based Pharmacokinetic Modeling Data}

PBPK Software Qualification. Simulated PK parameters for inhaled vilanterol 100 or $25 \mu \mathrm{g}$ were within the bioequivalence limit of quantification, which qualified the PBPK model built for vilanterol using Simcyp (Supplemental Table 11). Similarly, the AUC and $\mathrm{C}_{\max }$ ratios in the DDI simulations with ketoconazole were predicted within the bioequivalence limits (Supplemental Table 12). The observed geometric mean $\mathrm{C}_{\max }$ and AUC ratio for vilanterol-ketoconazole DDI were 0.90 and 1.90 , respectively, which compare well with the simulated $\mathrm{C}_{\max }$ and $\mathrm{AUC}$ ratios of 1.10 and 1.72 , respectively.

PBPK Model Qualification. The Simcyp-simulated and observed plasma concentration time curves for nemiralisib following $10 \mu \mathrm{g}$ i.v., oral $800 \mu \mathrm{g}$, and inhaled $1000 \mu \mathrm{g}$ administration are shown in Fig. 3. Simulated PK parameters were within acceptable bioequivalence limits of those observed clinically for all PBPK models (Supplemental Table 13). In addition, the mean Simcyp simulated oral bioavailability of $35 \%$ was within the range observed clinically following an $800 \mu \mathrm{g}$ oral dose at $33.7 \%-36.5 \%$ (Harrell et al., 2019). The in silico MPPD simulations predicted that $54 \%$ of the $1000 \mu \mathrm{g}(540 \mu \mathrm{g})$ were to be deposited in the lung. It is assumed that all of this drug deposited in the lung is absorbed. Further qualification of the inhaled nemiralisib model

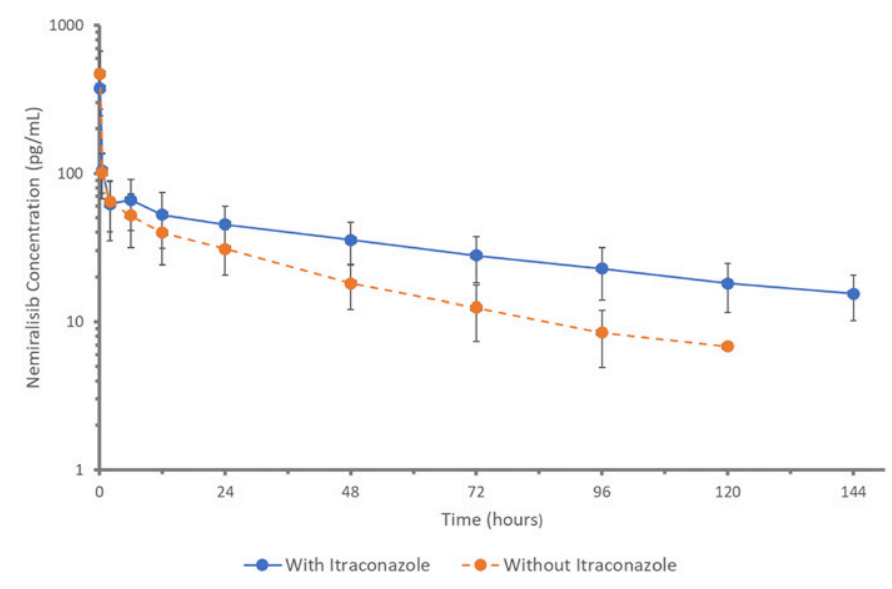

Fig. 2. Median nemiralisib concentration time plot from a clinical drug interaction study investigating the effect of oral itraconazole (a potent P4503A4 and P-gp inhibitor) on the pharmacokinetics of inhaled nemiralisib. 
TABLE 3

Summary of statistical analysis derived nemiralisib pharmacokinetic parameters following inhalation administration of nemiralisib alone and inhaled nemiralisib with oral itraconazole

\begin{tabular}{|c|c|c|c|c|c|c|}
\hline PK Parameter & Treatment & $N$ & $n$ & $\begin{array}{l}\text { Adjusted Geometric Mean } \\
\qquad(90 \% \mathrm{CI})\end{array}$ & $\begin{array}{c}\text { Ratio of Adjusted Geometric Means }(90 \% \text { CI), } \\
\text { Nemiralisib vs. Nemiralisib } \\
\text { with Itraconazole }\end{array}$ & $\begin{array}{l}\text { Mean } \\
\text { Squared } \\
\text { Error }\end{array}$ \\
\hline \multirow{2}{*}{$\begin{array}{l}\mathrm{AUC}_{0-\mathrm{inf}} \\
\quad\left(\mathrm{h}^{*} \mathrm{pg} / \mathrm{ml}\right)\end{array}$} & Nemiralisib $100 \mu \mathrm{g}$ single dose & 20 & 20 & $3199.0(2874.7,3559.9)$ & \multirow[t]{2}{*}{$2.01(1.81,2.22)$} & \multirow[t]{2}{*}{0.0253} \\
\hline & $\begin{array}{l}\text { Nemiralisib } 100 \mu \mathrm{g} \text { single dose with } \\
\text { itraconazole } 200 \mathrm{mg} \text { repeat dose }\end{array}$ & 20 & 14 & $6413.4(5695.9,7221.3)$ & & \\
\hline \multirow[t]{2}{*}{$\mathrm{C}_{\max }(\mathrm{pq} / \mathrm{ml})$} & Nemiralisib $100 \mu \mathrm{g}$ single dose & 20 & 20 & $478.7(416.4,550.3)$ & \multirow[t]{2}{*}{$0.802(0.690,0.933)$} & \multirow[t]{2}{*}{0.0762} \\
\hline & $\begin{array}{l}\text { Nemiralisib } 100 \mu \mathrm{g} \text { single dose with } \\
\text { itraconazole } 200 \mathrm{mg} \text { repeat dose }\end{array}$ & 20 & 20 & $384.0(334.0,441.4)$ & & \\
\hline
\end{tabular}

$\mathrm{AUC}_{0 \text {-inf }}$, area under the plasma concentration-time curve time zero (predose) extrapolated to infinite time; $\mathrm{CI}$, confidence interval; $\mathrm{C}_{\text {max }}$, maximum observed plasma concentration; $N$, number of subjects; $n$, number of evaluable subjects.

using simulated PK parameters to predict exposure after single inhaled doses of 500 and $100 \mu \mathrm{g}$ also demonstrated data within acceptable bioequivalence limits (data not shown).

Finally, the retrospective simulation of the clinical DDI study between nemiralisib and itraconazole showed good agreement between the simulated and observed data $\left(\mathrm{AUC}_{0 \text {-inf }}\right.$ ratio 2.25 and 2.01, respectively; Table 4).

PBPK Model Application. The PBPK Simcyp model predicted a weak drug interaction following coadministration of clarithromycin and erythromycin (simulated $\mathrm{AUC}_{0 \text {-inf }}$ ratio of 1.71 and 1.67 , respectively; Table 5). The simulated midazolam drug interaction study to assess the impact of nemiralisib on the exposure of sensitive P4503A4 substrates showed no change in the exposure of midazolam with steadystate dosing of nemiralisib $750 \mu \mathrm{g}$ once daily. A sensitivity analysis indicated that nemiralisib doses of greater than 100-times those used clinically would be needed to elicit a clinically relevant interaction with midazolam (Supplemental Fig. 1).

\section{Discussion}

According to U.S. Department of Health and Human Services Food and Drug Administration (2017b), moderate sensitive P4503A4 substrates are drugs whose $\mathrm{AUC}_{0 \text {-inf }}$ values increase by 2- to 5 -fold when coadministered with strong index $\mathrm{P} 4503 \mathrm{~A} 4$ inhibitors such as itraconazole. The 2.01-fold increase in $\mathrm{AUC}_{0 \text {-inf }}$ for nemiralisib when coadministered with itraconazole would therefore be classified as a moderate drug interaction, albeit at the very low end of this classification range. Importantly, the observed systemic exposure to itraconazole and its metabolite in the clinical study were in line with that observed following repeat doses of itraconazole at $200 \mathrm{mg}$ once daily (Backman et al., 1998), confirming that, based on systemic exposure, adequate drug levels of itraconazole were achieved for maximal inhibition of P4503A4. In comparison with the small fold changes to nemiralisib elicited by itraconazole, coadministration of itraconazole with midazolam (a sensitive P4503A4 substrate) resulted in an 11-fold change in midazolam $\mathrm{AUC}_{0 \text {-inf }}$ (Olkkola et al.,1994), which is substantially higher than that observed for nemiralisib. It is important to be able to extrapolate changes in systemic exposure observed in clinical drug interaction studies, not only to other potential comedications with increased or decreased potency but also to different routes, doses, regimens, or patient groups.

For this purpose, the PBPK platform Simcyp was qualified for inhalation using a published victim drug interaction for inhaled vilanterol with ketoconazole (Kempsford et al., 2013); the resultant simulated DDI for inhaled vilanterol was well within acceptable limits. In addition, the inhaled nemiralisib SimcypP PBPK model also accurately predicted the PK exposures observed in the clinical DDI between nemiralisib and itraconazole, reported herein.
Simcyp (version 17) does not have a specific inhalation application, but additional drug inputs can be built into the PBPK model using the Simcyp parameter estimation module, which, simply, requires an amount of drug and a rate constant (modeled from previous clinical studies). In this case, the amount of drug deposited in the lung was determined using MPPD, which is a sophisticated stand-alone tool to predict the total and regional human lung deposition from a given drug particle size profile (Asgharian et al., 1999, 2006; (Manojkumar et al., 2019)). Other lung/inhalation models are nicely summarized by Borghardt et al. (2015). Boger and Friden (2019) and Boger and Wigstrom (2018) describe multicompartment inhaled PBPK models that mechanistically describe processes such as deposition, mucociliary clearance, and dissolution. Andersen et al. (2000) incorporated computational fluid dynamic models to estimate the flux of inhaled chemicals in animal nasal cavities. However, at the time, it was not possible to combine any of these sophisticated approaches directly with Simcyp to investigate drug interaction liability.

The inhaled nemiralisib PBPK model was used to model prospective simulations with other P4503A4 inhibitors, including common comedications, in target patient populations. The $\mathrm{AUC}_{0 \text {-inf }}$ ratios of nemiralisib simulated by the PBPK model following coadministration with clarithromycin and erythromycin (1.71- and 1.67-fold, respectively) would be classified as weak interactions (U.S. Department of Health and Human Services Food and Drug Administration 2017b), with resultant nemiralisib systemic exposures (both AUC and $\mathrm{C}_{\max }$ ) considerably lower than exposures at the no adverse effect levels in animal toxicology studies (GSK, unpublished data). Any small changes in nemiralisib exposure, because of P4503A4 inhibition, were within the systemic overages calculated from long-term animal studies and of negligible significance for human administrations of nemiralisib up to $750 \mu \mathrm{g}$. The clinical drug interaction study between nemiralisib and itraconazole and subsequent extrapolations using the PBPK model indicated that dosing restrictions with other strong P4503A4 inhibitors, such as chronically administered macrolide antibiotics, are not required. As a result, in our opinion, it was concluded that erythromycin and clarithromycin (common comedications in a COPD or bronchiectasis indication) could be safely coadministered with nemiralisib in any subsequent clinical trials.

Although itraconazole is also a potent $\mathrm{P}$-gp inhibitor and nemiralisib is a P-gp substrate (data not shown), the clinical DDI study results do not demonstrate a significant $\mathrm{P}$-gp interaction as a result of $\mathrm{P}$-gp inhibition in the GI tract. Coadministration of nemiralisib with itraconazole did not increase nemiralisib $\mathrm{C}_{\max }$, which would be expected for a relevant P-gp mediated interaction. Furthermore, inspection of time concentration profiles (Fig. 2) shows the increase in nemiralisib AUC is driven by an increased elimination half-life in the inhibited state, which is consistent with a P4503A4 interaction. Any clinically significant DDI between 

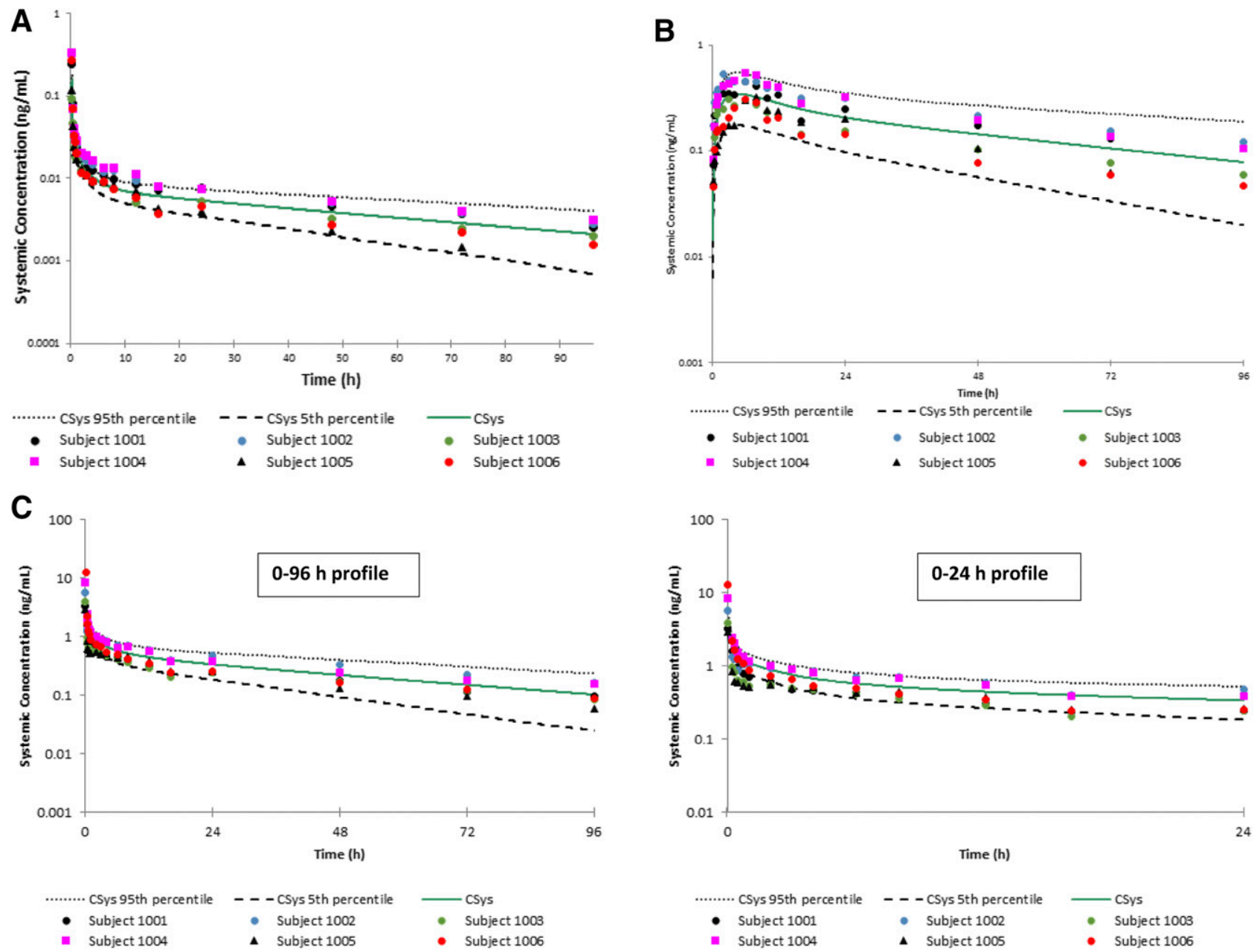

Fig. 3. Simcyp simulated (lines) and observed (symbols) plasma profiles after (A) $10 \mu \mathrm{g}$ intravenous dose, (B) $800 \mu \mathrm{g}$ oral dose, (C) $1000 \mu \mathrm{g}$ inhaled dose of nemiralisib in healthy volunteers.

nemiralisib and P-gp inhibitors is unlikely because nemiralisib has high permeability $(157 \mathrm{~nm} / \mathrm{s}$ in cell monolayers, unpublished data) and is well absorbed in vivo in human (Harrell et al., 2019).

Using the tiered approach described by the U.S. Department of Health and Human Services Food and Drug Administration (2017b), no further DDI investigations were required for P4501A2, 2C8, 2C9, 2C19, or 2D6 (i.e., $R_{1} \leq 1.02, R_{2} \leq 1.25$, or $R_{1}$ gut $\leq 11$ ) following the application of basic equations. The next tier of investigation (static mechanistic models) was, however, triggered for P4503A4 and P4502B6, in which "R value" thresholds were exceeded for direct inhibition on the GI tract

TABLE 4

Simulated and observed nemiralisib exposure ratio following inhalation administration in the presence of potent P4503A4 inhibitor itraconazole administered by the oral route

\begin{tabular}{lcc}
\hline & $\begin{array}{c}\text { Simulated Geometric Mean Ratio } \\
\text { (5th and 95th Centile) }\end{array}$ & $\begin{array}{c}\text { Observed Geometric Mean Ratio } \\
\text { (90\% CI of Ratio) (min-max) }\end{array}$ \\
\hline$N$ & $400\left(\begin{array}{c}20 \text { trials each with } \\
20 \text { subjects) } \\
1.0(0.99-1.01)\end{array}\right.$ & 20 \\
$\mathrm{C}_{\max }$ ratio & $2.25(1.66-3.36)$ & $0.802(0.690-0.933)$ \\
$\begin{array}{c}\mathrm{AUC} C_{0-\text { inf }} \\
\text { ratio }\end{array}$ & & $2.01(1.81-2.24)(1.48-3.24)^{a}$ \\
\hline
\end{tabular}

$\mathrm{AUC}_{0 \text {-inf }}$, area under the plasma concentration-time curve time zero (predose) extrapolated to infinite time; $\mathrm{CI}$, confidence interval; $\mathrm{C}_{\max }$, maximum observed plasma concentration.

${ }^{a} N=14$.
(P4503A4) and MDI (P4503A4 and P4502B6). Static mechanistic models indicated no DDI risk associated with $\mathrm{P} 4502 \mathrm{~B} 6$ but could not be used to discharge the P4503A4 DDI perpetrator risks, which, therefore, required escalation to the next tier of investigation involving the application of a PBPK model. Application of the inhaled nemiralisib PBPK model, developed and applied herein, indicated no risk of a P4503A4 perpetrator DDI for nemiralisib. A sensitivity analysis using midazolam revealed that doses well in excess of likely nemiralisib clinical doses (approximately 100-fold) would be required to elicit a significant drug interaction ( $\geq 1.25$-fold) as a result of P4503A4 metabolism-dependent inhibition. A specific investigation into the potential for a DDI between nemiralisib and theophylline, a sensitive narrow therapeutic index P4503A4 substrate that had been identified as a common comedication in exacerbating patients, indicated a negligible risk using static mechanistic models. It is of significant note that without the PBPK model for nemiralisib, a P4503A4 perpetrator clinical drug interaction study would be needed as part of the clinical development plan. The effort to develop, validate, and apply such models is justified through reductions in unnecessary clinical trials, fewer subjects exposed to investigational drugs, and future cost savings.

The impact of nemiralisib on drug transporters P-gp, BCRP, OAT1, OAT3, OATP1B1, OATP1B3, OCT2, MATE1, and MATE2-K was also studied in vitro. With the exception of OAT1 and OAT3, nemiralisib was a potent inhibitor of all these transporters, with $\mathrm{IC}_{50}$ values ranging 0.0260-3.03 $\mu \mathrm{M}$. In four out of the nine values, the potency with 
TABLE 5

Prospective drug interaction predictions with P4503A4 inhibitors clarithromycin and erythromycin

\begin{tabular}{|c|c|c|}
\hline & $\begin{array}{l}\text { Simulated Ratio with } \\
\text { Clarithromycin }\end{array}$ & $\begin{array}{l}\text { Simulated Ratio with } \\
\text { Erythromycin }\end{array}$ \\
\hline $\begin{array}{l}\text { Simulated } \\
\text { individuals }\end{array}$ & 1000 & 1000 \\
\hline $\begin{array}{l}\text { Reference for study } \\
\text { design }\end{array}$ & Gorski et al. 1998 & Olkkola et al. 1994 \\
\hline $\begin{array}{l}\text { Simulated study } \\
\text { design }\end{array}$ & $\begin{array}{c}500 \mathrm{mg} \text { twice a day for } \\
7 \text { days and } 4 \mathrm{mg} \text { midazolam } \\
\text { on day } 7 \text { ( } 2 \mathrm{~h} \text { after the first } \\
\text { dose of clarithromycin) }\end{array}$ & $\begin{array}{l}500 \mathrm{mg} \text { erythromycin three } \\
\text { times a day for } 1 \mathrm{wk} \text { and } \\
15 \mathrm{mg} \text { midazolam on day } 6 \\
\text { ( } 2 \mathrm{~h} \text { after second dose of } \\
\text { the day) }\end{array}$ \\
\hline $\begin{array}{l}\text { Age range for } \\
\text { simulated } \\
\text { individuals }^{a}\end{array}$ & $21-62$ & $21-62$ \\
\hline $\begin{array}{l}\text { Simulated gender } \\
\text { ratio }^{a}\end{array}$ & Males & Males \\
\hline $\begin{array}{l}\mathrm{AUC}_{0 \text {-inf }} \text { geometric } \\
\text { mean ratio ( } 5 \text { th } \\
\text { and } 95 \text { th centile) }\end{array}$ & $1.71(1.27-2.48)$ & $1.67(1.22-2.40)$ \\
\hline
\end{tabular}

$\mathrm{AUC}_{0 \text {-inf, }}$ area under the plasma concentration-time curve time zero (pre-dose) extrapolated to infinite time.

${ }^{a}$ Demographics based on NCT03398421.

nemiralisib was higher than that observed for the positive control (Supplemental Table 9). The potential for nemiralisib to alter the clinical drug exposure of comedications, whose disposition is dependent on these drug transporters, was assessed using basic equations as described by the European Medicines Agency (2012) and U.S. Department of Health and Human Services Food and Drug Administration (2017b). Despite the high in vitro potencies, no systemic DDI risk was predicted up to maximum nemiralisib inhalation dose of $750 \mu \mathrm{g}$.

Interpretation of the nemiralisib in vitro inhibition data support the notion that the risk of perpetrator drug interactions is negligible for lowdose inhaled molecules. This is nicely illustrated by the data for MATE1 and MATE2-K, in which $\mathrm{IC}_{50}$ values of 0.0260 and $0.0545 \mu \mathrm{M}$ were $>100$-fold more potent than the cimetidine positive control, making nemiralisib one of the most potent MATE inhibitors ever described. Despite this, no threshold of concern was triggered when applying approaches recommended in regulatory guidelines. Although the risk of perpetrator drug interactions can be considered negligible for low-dose inhalation molecules, $\mathrm{P} 450$ and transporter inhibition data should still be contextualized against clinical exposures using the framework outlined in guidance documents. It is prudent, therefore, to generate such inhibition data ahead of studies in patient populations, even for lowdose inhalation molecules, to defend the inclusion of common comedications in clinical trial design and avoid any potential delay to the clinical trial approval process. Corresponding victim drug interaction risks cannot be discharged using the same "low-dose inhalation" argument when a variety of factors come into play. These include the type of dose limiting toxicity, potency, therapeutic index, competing pathways, and enzyme kinetics. In the case of nemiralisib, a P4503A4 victim clinical drug interaction study was needed to support the inclusion of macrolide antibiotics in special patient groups. Regulatory authorities expect to see a consideration of both victim and perpetrator risks aligned to regulatory guidance, and the holistic approach used to assess the DDI risk with inhaled nemiralisib, in our view, addressed these regulatory requirements.

In conclusion, using an integrated in silico, in vitro, and clinical approach, we have shown that any clinically significant DDIs with nemiralisib are unlikely and that a range of common comedications can be allowed safely into patient clinical study designs. Though inhaled drugs are expected to have low systemic exposure, it remains prudent to follow regulatory guidance, including the tiered approach for perpetrator interactions. An integrated DDI assessment should be conducted for inhaled molecules and should include consideration of any comedications relevant for the specific therapeutic area, in vitro perpetrator liabilities, and victim investigations.

\section{Acknowledgments}

The authors would like to thank Azmina Mather, who was the GSK study monitor for the drug transporter assays, and Jon Robertson, GSK, for reviewing the statistical analyses reported in the manuscript.

\section{Authorship Contributions}

Participated in research design: Patel, Wilson, Harrell, Taskar, Taylor, Tracey, Cahn, Marotti.

Conducted experiments: Taskar, Tracey, Taylor.

Performed data analysis: Patel, Wilson, Taskar, Taylor, Tracey, Riddell, Marotti.

Wrote or contributed to the writing of the manuscript: All authors.

\section{References}

Andersen M, Sarangapani R, Gentry R, Clewell H, Covington T, and Frederick CB (2000) Application of a hybrid CFD-PBPK nasal dosimetry model in an inhalation risk assessment: an example with acrylic acid. Toxicol Sci 57:312-325.

Asgharian B, Miller FJ, and Subramaniam RP (1999) Dosimetry software to predict particle deposition in humans and rats. CIIT Activities 19:1-6.

Asgharian B, Price OT, and Hofmann W (2006) Prediction of particle deposition in the human lung using realistic models of lung ventilation. J Aerosol Sci 37:1209-1221.

Backman JT, Kivistö KT, Olkkola KT, and Neuvonen PJ (1998) The area under the plasma concentration-time curve for oral midazolam is 400 -fold larger during treatment with itraconazole than with rifampicin. Eur J Clin Pharmacol 54:53-58.

Begg M, Wilson R, Hamblin JN, Montembault M, Green J, Deans A, Amour A, Worsley S, Fantom K, Cui Y, et al. (2019) Relationship between pharmacokinetics and pharmacodynamic responses in healthy smokers informs a once-daily dosing regimen for nemiralisib. J Pharmacol Exp Ther 369:337-344.

Bloomer J, Derimanov G, Dumont E, Ellens H, and Matheny C (2013) Optimizing the in vitro and clinical assessment of drug interaction risk by understanding co-medications in patient populations. Expert Opin Drug Metab Toxicol 9:737-751.

Boger E and Fridén M (2019) Physiologically based pharmacokinetic/pharmacodynamic modeling accurately predicts the better bronchodilatory effect of inhaled versus oral salbutamol dosage forms. J Aerosol Med Pulm Drug Deliv 32:1-12.

Boger E and Wigström O (2018) A partial differential equation approach to inhalation physiologically based pharmacokinetic modeling. CPT Pharmacometrics Syst Pharmacol 7:638-646.

Borghardt JM, Weber B, Staab A, and Kloft C (2015) Pharmacometric models for characterizing the pharmacokinetics of orally inhaled drugs. AAPS $J$ 17:853-870.

Cahn A, Hamblin JN, Begg M, Wilson R, Dunsire L, Sriskantharajah S, Montembault M, Leemereise CN, Galinanes-Garcia L, Watz H, et al. (2017) Safety, pharmacokinetics and doseresponse characteristics of GSK2269557, an inhaled PI3K $\delta$ inhibitor under development for the treatment of COPD. Pulm Pharmacol Ther 46:69-77.

Down K, Amour A, Baldwin IR, Cooper AW, Deakin AM, Felton LM, Guntrip SB, Hardy C, Harrison ZA, Jones KL, et al. (2015) Optimization of novel indazoles as highly potent and selective inhibitors of phosphoinositide 3-kinase $\delta$ for the treatment of respiratory disease. $\mathrm{J} \mathrm{Med}$ Chem 58:7381-7399.

European Medicines Agency(2012) Guideline on the Investigation of Drug Interactions, CPMP/ EWP/560/95/Rev. 1 Corr. 2. European Medicines Agency, London, UK.

Foisy MM, Yakiwchuk EMK, Chiu I, and Singh AE (2008) Adrenal suppression and Cushing's syndrome secondary to an interaction between ritonavir and fluticasone: a review of the literature. HIV Med 9:389-396.

Gorski JC, Jones DR, Haehner-Daniels BD, Hamman MA, O'Mara EM Jr., and Hall SD (1998) The contribution of intestinal and hepatic CYP3A to the interaction between midazolam and clarithromycin. Clin Pharmacol Ther 64:133-143.

Harrell AW, Wilson R, Man YL, Riddell K, Jarvis E, Young G, Chambers R, Crossman L, Georgiou A, Pereira A, et al. (2019) An innovative approach to characterize clinical ADME and pharmacokinetics of the inhaled drug nemiralisib using an intravenous microtracer combined with an inhaled dose and an oral radiolabel dose in healthy male subjects. Drug Metab Dispos 47:1457-1468.

Ke AB, Zamek-Gliszczynski MJ, Higgins JW, and Hall SD (2014) Itraconazole and clarithromycin as ketoconazole alternatives for clinical CYP3A inhibition studies. Clin Pharmacol Ther 95: 473-476.

Kempsford R, Allen A, Bal J, Rubin D, and Tombs L (2013) The effect of ketoconazole on the pharmacokinetics and pharmacodynamics of inhaled fluticasone furoate and vilanterol trifenatate in healthy subjects. Br J Clin Pharmacol 75:1478-1487.

Lawrence SK, Nguyen D, Bowen C, Richards-Peterson L, and Skordos KW (2014) The metabolic drug-drug interaction profile of Dabrafenib: in vitro investigations and quantitative extrapolation of the P450-mediated DDI risk. Drug Metab Dispos 42:1180-1190.

Manojkumar N, Srimuruganandam B, and Shiva Nagendra SM (2019) Application of multiple-path particle dosimetry model for quantifying age specified deposition of particulate matter in human airway. Ecotoxicol Environ Saf 168:241-248.

Olkkola KT, Backman JT, and Neuvonen PJ (1994) Midazolam should be avoided in patients receiving the systemic antimycotics ketoconazole or itraconazole. Clin Pharmacol Ther 55:481-485. Shebley M, Sandhu P, Emami Riedmaier A, Jamei M, Narayanan R, Patel A, Peters SA, Reddy VP, Zheng M, de Zwart L, et al. (2018) Physiologically based pharmacokinetic model qualification and reporting procedures for regulatory submissions: a consortium perspective. Clin Pharmacol Ther 104:88-110. 
Sirmans SM, Pieper JA, Lalonde RL, Smith DG, and Self TH (1988) Effect of calcium channel blockers on theophylline disposition. Clin Pharmacol Ther 44:29-34.

Somers GI, Lindsay N, Lowdon BM, Jones AE, Freathy C, Ho S, Woodrooffe AJM, Bayliss MK, and Manchee GR (2007) A comparison of the expression and metabolizing activities of phase I and II enzymes in freshly isolated human lung parenchymal cells and cryopreserved human hepatocytes. Drug Metab Dispos 35:1797-1805.

Stringer KA, Mallet J, Clarke M, and Lindenfeld JA (1992) The effect of three different oral doses of verapamil on the disposition of theophylline. Eur J Clin Pharmacol 43:35-38.

Tjia JF, Colbert J, and Back DJ (1996) Theophylline metabolism in human liver microsomes: inhibition studies. J Pharmacol Exp Ther 276:912-917.

U.S. Department of Health and Human Services Food and Drug Administration (2006) Drug Interaction Studies - Study Design, Data Analysis and Implications for Dosing and Labelling, Draft Guidance, U.S. Department of Health and Human Services, Washington, DC.

U.S. Department of Health and Human Services Food and Drug Administration (2017a) Clinical Drug Interaction Studies - Study Design, Data Analysis, and Clinical Implications, Guidance for Industry, Draft Guidance, U.S. Department of Health and Human Services, Washington, DC.
U.S. Department of Health and Human Services Food and Drug Administration (2017b) In Vitro Metabolism and Transport-Mediated Drug-Drug Interaction Studies, Guidance for Industry, Draft Guidance, U.S. Department of Health and Human Services, Washington, DC.

Wilson R, Jarvis E, Montembault M, Hamblin JN, Hessel EM, and Cahn A (2018) Safety, tolerability, and pharmacokinetics of single and repeat doses of nemiralisib administered via the Ellipta dry powder inhaler to healthy subjects. Clin Ther 40:1410-1417.

Wilson R, Templeton A, Leemereise C, Eames R, Banham-Hall E, Hessel EM, and Cahn A (2019) Safety, tolerability, and pharmacokinetics of a new formulation of nemiralisib administered via a dry powder inhaler to healthy individuals. Clin Ther 41:1214-1220.

Address correspondence to: Aarti Patel, Drug Metabolism and Pharmacokinetics, GlaxoSmithKline R\&D, United Kingdom. E-mail: aarti.2.patel@gsk.com 